\title{
Investigation of the topographical differences in somatosensory sensitivity of the human nasal mucosa*
}

Mandy Scheibe, Annika Schmidt, Thomas Hummel

Smell \& Taste Clinic, Department of Otorhinolaryngology, University of Dresden Medical School, Dresden, Germany
Rhinology 50: 290-293, 2012

DOI:10.4193/Rhino11.224

*Received for publication:

October 23, 2011

Accepted: April 25, 2012

\section{Summary}

Background: Previous investigations in humans suggest topographical differences in intranasal trigeminal chemosensitivity with the highest sensitivity in the anterior part. The present study aimed to investigate whether different sites in the human nasal mucosa react differently to unspecific electrical stimuli.

Methology: Participants were 50 young, healthy volunteers (24 men, 26 women; age 22-38 years). Detection and pain threshold of electrical trigeminal stimuli were investigated at 5 different sites: anterior septum, posterior septum, inferior turbinate, middle turbinate and anterior lateral wall.

Results: In healthy subjects, a significantly higher trigeminal sensitivity was found at the anterior parts of the nose compared to the posterior part. There was a similar distribution pattern of the sensitivity for detection and pain thresholds.

Conclusions: Results suggest that there are consistent topographical differences in the arrangement of trigeminal receptors of the human nasal cavity; highest somatosensory sensitivity seems to be located in the anterior part. This finding is compatible with the idea that the trigeminal system acts as a sentinel of the human airways with regard to toxic agents.

Key words: trigeminal sensitivity, electrical stimuli, nose

\section{Introduction}

Intranasal somatosensory sensitivity is mediated by trigeminal afferents. Until a short time ago it was assumed that irritants activate free endings of the trigeminal nerves directly in an unspecific way. Today specific receptors of the trigeminal system have been identified, for example the acid-sensitive ionic channels (ASIC receptors) ${ }^{(1)}$ and the capsaicin receptor/vanilloid receptor-1 (TRPV1) ${ }^{(2,3)}$. They are activated by chemosensory as well as by thermal stimuli. Trigeminally mediated sensations include burning, stinging, prickling, sharpness, warmth, and cooling ${ }^{(4,5)}$.

The intranasal sensitivity has different functions. First, the trigeminal system is intimately involved in the perception of odours because most odours activate both, the olfactory and the trigeminal nerves ${ }^{(6,7)}$. Interactions between olfaction and the trigeminal system have been shown in many previous studies. For example, in anosmic subjects the trigeminal sensitivity is also reduced ${ }^{(8,9)}$; conversely, loss of trigeminal function seems also to result in a reduced olfactory function ${ }^{(10)}$. Second, the intranasal trigeminal system seems to be highly significant for the protection of the airways against toxic irritants, because the nasal mucosa is the first tissue, which has contact with potentially toxic agents carried by the inhaled airstream. Intranasal trigeminal activation generates protective reflexes such as sneezing or apnea to prevent noxious substances from entering the respiratory system ${ }^{(4,11,12)}$. Third, another important role is the perception of nasal airflow. For example, menthol 
sensitizes trigeminal receptors like the cold receptor TRPM8 (13), providing the feeling of a 'free nose' without decongestive effect. The reason for this impression is an increased sensitivity for the nasal airflow ${ }^{(14,15)}$. On the other hand, local anaesthesia produces a sensation of nasal obstruction without a major effect on nasal resistance to airflow ${ }^{(16)}$.

Numerous studies indicate that the respiratory mucosa is an inhomogeneous tissue in terms of the sensitivity to irritants. Previous works in animals and humans suggest that trigeminal receptors are not equally spread throughout the nasal cavity. Different methods for investigations were used, such as histochemical studies ${ }^{(17,18)}$, electrophysiological recordings from the respiratory epithelium ${ }^{(19-21)}$ or recordings of cortical event-related potentials ${ }^{(22)}$ and intensity ratings ${ }^{(22)}$ following stimulation at various areas of the nasal cavity. All of them found the highest trigeminal sensitivity in the anterior part of the nasal cavity. Furthermore, Konstantinidis et al., suggest that nasal anatomy plays a significant role in determining interindividual differences in the sensitivity to trigeminal stimuli ${ }^{(23)}$.

Knowledge of the trigeminal sensitivity in the human nasal mucosa seems to be important in the clinical daily routine. For example, in surgery it is necessary to preserve mucosa with high trigeminal function. In patients with the so-called 'empty nose syndrome' (24), next to alteration of the airflow inside the nose, the loss of trigeminal receptors also contributes to the impression of a 'congested nose.'

The aim of the present study was to investigate the trigeminal sensitivity at different sites at the human nasal mucosa again, now in contrast to former studies using unspecific direct electrical stimuli. Using suprathreshold electrical stimuli all trigeminal receptors (thermal, tactile, chemosensory etc.) are activated. Thus, the present work is about results from passive somatosensory stimulation.

\section{Materials and methods}

Study design

Fifty healthy volunteers (24 men, 26 women; age 22 - 38 years) participated. Participants were given detailed information about all testing procedures and written informed consent was provided by all subjects prior to the study. All measurements were performed according to the Declaration of Helsinki. The study design was approved by the Ethics Committee of the University of Dresden Medical School (EK number 88052006).

At first the subjects' medical history was obtained and nasal endoscopy was performed by an ENT specialist (MS). After that olfactory function was screened by means of an odour identification test, separately for each nostril ('Sniffin' Sticks' ${ }^{(25)}$ ) ascertaining normosmia. Here the subjects' task is to identify 12 odours presented to either nostril in a randomized sequence; the sum of correctly identified odours is the score, which relates to olfactory sensitivity. For screening of the chemosensory trigeminal function all participants also underwent the 'lateralization test' as previously described ${ }^{(26,27)}$; within this paradigm menthol, ethanol, and cinnamon aldehyde are presented 20 times each to the left or right nostril with the subjects indicating which side had been stimulated. Because lateralization is a function of trigeminal sensitivity the scores from this screening test relate to trigeminal sensitivity. None of the subjects reported breathing difficulties, acute nasal allergies, or acute rhinitis; nasal endoscopy revealed no pathology.

Both detection and pain threshold of trigeminal stimuli of the subjects were investigated with electrical stimuli applied with a spherical electrode (Figure 1; PowerLab ADInstruments, Spechbach, Germany). Ascending stimulus intensities beginning at $0.5 \mathrm{~mA}$ were used; stimulus intensity was increased by $0.05 \mathrm{~mA}$ until subjects reliably indicated detection of the stimuli. From then on intensity was increased by $0.1 \mathrm{~mA}$ until participants indicated that the stimulus was painful. The stimulus duration was constant at $0.05 \mathrm{~ms}$. Recordings were performed at the following five different sites of the nasal mucosa: anterior septum, posterior septum, inferior turbinate, middle turbinate, and anterior lateral nasal wall (Figure 1). To keep the spherical electrode in place, it was attached to clips mounted on a frame similar to lensless glasses. The position of the electrode's tip was controlled by nasal endoscopy. The sequence of measurements at the five different locations was randomized across all subjects. In an individual subject measurements were always carried out in the same nostril.

\section{Statistical analyses}

Results were analyzed using SPSS vs.17 (SPSS Inc., Chicago, IL, USA) using analyses of variance for repeated measures. F-values are presented together with degrees of freedom [in brackets] followed by the $p$ value). To investigate differences between stimulation sites t-tests for paired samples were used (degrees of freedom Bonferroni-corrected). The level of significance was set at 0.05 .

\section{Results}

As ascertained by means of the 'Sniffin' Sticks' screening test all subjects exhibited normosmia ( $M=11.0, S D=0.8)$. Similarly, when screening trigeminal function, using the lateralization test, subjects showed a normal trigeminal sensitivity (menthol: $M=17.5, S D=2.1$; ethanol: $M=15.2, S D=2.7$; cinnamon aldehyde: $M=17.1, S D=3.2$ ).

The detection thresholds for trigeminal stimuli were significantly different at the various sites of stimulation of the nasal mucosa $(F[4,232]=34.9, p<0.001)$. Anterior parts of the nose, namely, the anterior septum, the inferior turbinate and the anterior lateral nasal wall showed significantly $(p<0.001)$ higher trigeminal sensitivity than posterior parts, namely, the posterior septum and the middle turbinate. In addition, the 3 

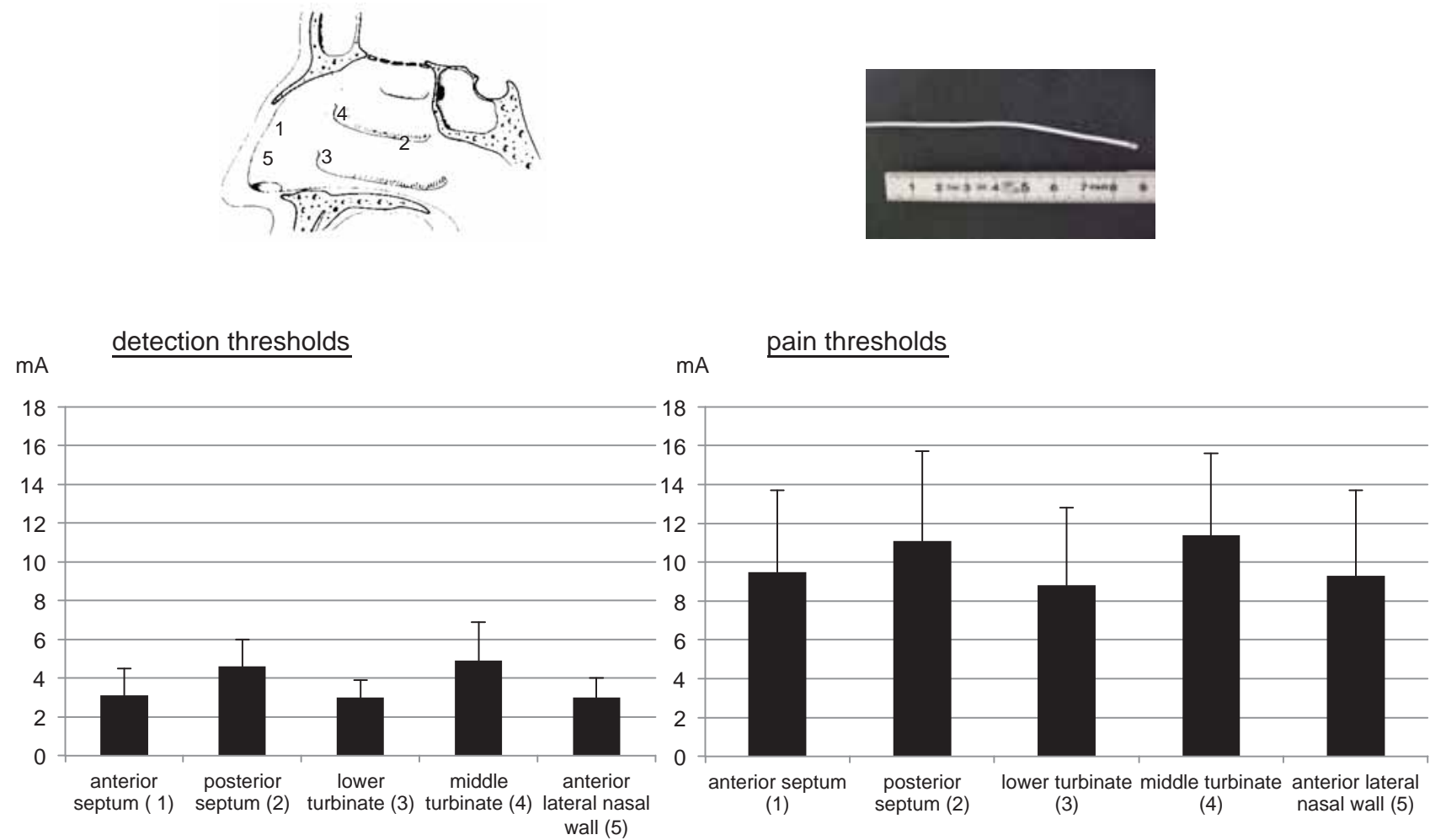

Figure 1. Mean values of the detection (left) and pain thresholds (right) of healthy subjects ( $n=50)$ in response to electrical stimuli applied to different sites at the human nasal mucosa (error bars indicate standard errors of means). The insert top left indicates the location of the five recording sites. The insert top right is a photo of the stimulation probe.

anterior stimulation sites were not significantly different from each other; also, the two posterior stimulation sites did not differ in terms of sensitivity ( $p=1.0)$.

A similar distribution pattern was found for pain thresholds ( $F$ $[4,232]=9.59, p<0.001)$; pain thresholds from the 5 sites followed the pattern as had been seen with detection thresholds (see above; $p<0.01$ ) with the only exception that the anterior septum did not differ significantly from all other stimulation sites $(p>0.05)$.

\section{Discussion}

Results of the present study suggest that humans exhibit topographical differences in somatosensory sensitivity and that in this regard the respiratory mucosa is an inhomogeneous tissue. A high sensitivity seems to be present in the anterior part of the nasal cavity. Importantly, in the present study unspecific electrical stimuli were used.

The present results are in line with previous research, where other techniques were utilized. Using cortical event-related potentials and intensity ratings to mechanical (air puffs) and chemosensory $\left(\mathrm{CO}_{2}\right)$ stimuli were investigated by Frasnelli et al., (22) at the anterior and posterior part of the nose. The authors found the highest chemosensory sensitivity in the anterior portion, the highest mechanical sensitivity in the back of the nose. The intensity of the chemosensory irritant was also strongest at the anterior nasal cavity (but see also ${ }^{(28)}$ ).

Differences in the distribution of intranasal trigeminal receptors in humans have also been investigated using an electrophysiological measure of trigeminally induced activation ${ }^{(20,21)}$, the negative mucosa potential (NMP). It was found ${ }^{(20,21)}$ that the nasal septum is more sensitive to stimulation with irritants, compared to other locations within the nasal mucosa like the olfactory cleft and the inferior turbinate or the middle turbinate and the floor of the nasal cavity. A recent study from Meusel et al., ${ }^{(19)}$ also showed topographical differences in the responsiveness of the nasal mucosa to irritants which supports the present findings.

Using histochemical methods, Lee et al., investigated regional differences of the rat's nasal intraepithelial sensory nerve fibers. They reported a higher nerve fiber density in anterior than posterior regions of the nose. Their conclusion was that this pattern relates to the detection of noxious stimuli ${ }^{(17)}$. In addition, so-called solitary chemosensory cells (SCCs), which seem to have a supporting function of the intranasal trigeminal system, were found in the anterior parts of the nose. Here, 
it may also be speculated that this distribution would also contribute to the relatively high sensitivity in this area ${ }^{(18,29)}$.

\section{Conclusion}

Results from the present study, in line with previous research, suggest that there are topographical differences in the arrangement of the intranasal somatosensory sensitivity with the highest sensitivity in the anterior part of the nasal cavity. The most important area for monitoring inhaled air seems to be the anterior section of the nose, which is compatible with the idea that the trigeminal system acts as a sentinel of the human airways.

\section{Authorship contribution}

All authors had full access to all the data in the study and take responsibility for the integrity of the data and the accuracy of the data analysis.

\section{Conflict of interest}

None of the authors declares a conflict of interest.

\section{References}

1. Waldmann R, Champigny G, Bassilana F, Heurteaux C, Lazdunski M. A proton-gated cation channel involved in acid-sensing. Nature. 1997; 13; 386: 173-177.

2. Caterina MJ, Schumacher MA, Tominaga $M$, Rosen TA, Levine JD, Julius D. The capsaicin receptor: a heat-activated ion channel in the pain pathway. Nature. 1997; 389: 816824.

3. Trevisani M, Smart D, Gunthorpe MJ, Tognetto M, Barbieri M, Campi B, et al. Ethanol elicits and potentiates nociceptor responses via the vanilloid receptor-1. Nat Neurosci. 2002; 5: 546-551.

4. Stevens JC, Cain WS. Aging and the perception of nasal irritation. Physiol Behav. 1986; 37: 323-328.

5. Laska M, Distel H, Hudson R. Trigeminal perception of odorant quality in congenitally anosmic subjects. Chem Senses. 1997; 22: 447-456.

6. Hummel T, Livermore A. Intranasal chemosensory function of the trigeminal nerve and aspects of its relation to olfaction. Int Arch Occ Env Health. 2002; 75: 305-313.

7. Doty RL, Brugger WE, Jurs PC, Orndorff MA, Snyder PJ, Lowry LD. Intranasal trigeminal stimulation from odorous volatiles: psychometric responses from anosmic and normal humans. Physiol Behav. 1978; 20: 175-185.

8. Hummel T. Assessment of intranasal trigeminal function. Int J Psychophysiol. 2000; 36: 147-155.

9. Frasnelli J, Schuster B, Hummel T. Interactions between olfaction and the trigeminal system: what can be learned from olfactory loss. Cereb Cortex. 2007; 17: 2268-2275.

10. Husner A, Frasnelli J, Welge-Lussen A, Reiss G, Zahnert T, Hummel T. Loss of trigeminal sensitivity reduces olfactory function. The Laryngoscope. 2006; 116: 1520-1522.

11. Kendal-Reed M, Walker JC, Morgan WT. Investigating sources of response variability and neural mediation in human nasal irritation. Indoor Air. 2001; 11: 185-191.

12. Sant'Ambrogio G, Tsubone H, Sant'Ambrogio FB. Sensory information from the upper air- way: role in the control of breathing. Respir Physiol. 1995; 102: 1-16.

13. Peier AM, Moqrich A, Hergarden AC, Reeve AJ, Andersson DA, Story GM, et al. A TRP channel that senses cold stimuli and menthol. Cell. 2002; 108: 705-715.

14. Nishino T, Tagaito Y, Sakurai Y. Nasal inhalation of I-menthol reduces respiratory discomfort associated with loaded breathing. Am J Respir Crit Care Med. 1997; 156: 309313.

15. Eccles $R$, Jones AS. The effect of menthol on nasal resistance to air flow. J Laryngol Otol. 1983; 97: 705-709.

16. Jones AS, Crosher R, Wight RG, Lancer JM, Beckingham E. The effect of local anaesthesia of the nasal vestibule on nasal sensation of airflow and nasal resistance. Clin Otolaryngol Allied Sci. 1987; 12: 461-464.

17. Lee SH, Iwanaga T, Hoshi O, Adachi I, Fujita T. Regional differences of CGRPimmunoreactive nerve fibers in nasal epithelium of the rat. Arch Histol Cytol. 1995; 58: 117-126.

18. Finger TE, Bottger B, Hansen A, Anderson KT, Alimohammadi H, Silver WL. Solitary chemoreceptor cells in the nasal cavity serve as sentinels of respiration. Proc Natl Acad Sci USA. 2003; 100: 8981-8986.

19. Meusel T, Negoias S, Scheibe M, Hummel T. Topographical differences in distribution and responsiveness of trigeminal sensitivity within the human nasal mucosa. Pain. 2010; 151: 516-521.

20. Scheibe $M$, Zahnert T, Hummel T. Topographical differences in the trigeminal sensitivity of the human nasal mucosa. Neuroreport. 2006; 17: 1417-1420.

21. Scheibe M, van Thriel C, Hummel T. Responses to trigeminal irritants at different locations of the human nasal mucosa. Laryngoscope. 2008; 118: 152-155.

22. Frasnelli J, Heilmann S, Hummel T. Responsiveness of human nasal mucosa to trigeminal stimuli depends on the site of stimulation. Neurosci Lett. 2004; 362: 65-69.

23. Konstantinidis I, Gartz I, Gerber JC, Reden J, Hummel T. Anatomy of the nasal cavity determines intranasal trigeminal sensitivity.
Rhinology. 2010; 48: 18-22.

24. Moore GF, Freeman TJ, Ogren FP, Yonkers AJ. Extended follow-up of total inferior turbinate resection for relief of chronic nasal obstruction. Laryngoscope. 1985; 95: $1095-$ 1099.

25. Kobal G, Klimek L, Wolfensberger M, Gudziol $\mathrm{H}$, Temmel A, Owen CM, et al. Multicenter investigation of 1,036 subjects using a standardized method for the assessment of olfactory function combining tests of odor identification, odor discrimination, and olfactory thresholds. Eur Arch Otorhinolaryngol. 2000; 257: 205-211.

26. Wysocki CJ, Cowart BJ, Radil T. Nasal trigeminal chemosensitivity across the adult life span. Percept Psychophys. 2003; 65: 115122.

27. Frasnelli J, Hummel T. Intranasal trigeminal threshold in healthy subjects. Environ Toxicol Phar. 2005; 19: 575-580.

28. Melzner J, Bitter T, Guntinas-Lichius O, Gottschall R, Walther M, Gudziol H. Comparison of the orthonasal and retronasal detection thresholds for carbon dioxide in humans. Chem Senses. 2011; 36: 435-441.

29. Hansen. A, Witt. M, Hummel. T. Unconventional neurons in the nasal cavity of humans. Chem Senses. 2005; 30: A180.

Thomas Hummel, M.D.

Smell \& Taste Clinic

Department of Otorhinolaryngology

University of Dresden Medical School

Fetscherstr. 74

01307 Dresden

Germany

Tel: +49-351-458-4189

Fax: +49-351-458-7370

E-mail:

thummel@mail.zih.tu-dresden.de 